\title{
Massive MIMO as Enabler for Communications with Drone Swarms
}

\author{
Prabhu Chandhar, Danyo Danev and Erik G. Larsson \\ Conference Publication
}

\section{Tweet}

N.B.: When citing this work, cite the original article.

Original Publication:

Prabhu Chandhar, Danyo Danev and Erik G. Larsson, Massive MIMO as Enabler for Communications with Drone Swarms, 2016 INTERNATIONAL CONFERENCE ON UNMANNED AIRCRAFT SYSTEMS (ICUAS), 2016. (), pp.347-354.

http://dx.doi.org/10.1109/ICUAS.2016.7502655

Copyright: www.iee.org

Postprint available at: Linköping University Electronic Press

http://urn.kb.se/resolve?urn=urn:nbn:se:liu:diva-129713

I..U 


\title{
Massive MIMO as Enabler for Communications with Drone Swarms
}

\author{
Prabhu Chandhar, Danyo Danev, and Erik G. Larsson \\ Dept. of Electrical Eng. (ISY), Linköping University, 58183 Linköping, Sweden \\ Email: \{prabhu.c, danyo.danev, erik.g.larsson\}@liu.se
}

\begin{abstract}
Massive multiple-input multiple-output (MIMO) is an emerging technology for mobile communications, where a large number of antennas are employed at the base station to simultaneously serve multiple single-antenna terminals with very high capacity. In this paper, we study the potentials and challenges of utilizing massive MIMO for unmanned aerial vehicles (UAVs) communication. We consider a scenario where multiple single-antenna UAVs simultaneously communicate with a ground station (GS) equipped with a large number of antennas. Specifically, we discuss the achievable uplink (UAV to GS) capacity performance in the case of line-of-sight (LoS) conditions. We also study the type of antenna polarization that should be used in order to maintain a reliable communication link between the GS and the UAVs. The results obtained using a realistic geometric model show that massive MIMO is a potential enabler for high-capacity UAV networks.

Index Terms-unmanned aerial vehicles, massive MIMO.
\end{abstract}

\section{INTRODUCTION}

In recent years, the use of unmanned aerial vehicles (UAVs), also known as drones, particularly micro UAVs, for both civilian and military applications is increasing worldwide due to their ability to perform multiple functions autonomously or with human control. In the next few years a large number of UAVs will coexist which would require a high-throughput network for communication [1], [2]. However, the communication between the ground station (GS) and the UAVs involves many challenges. First, in these applications, since the UAVs are usually equipped with highresolution cameras, delivering of high-resolution images and videos to the GS requires high speed communication. The main challenge here is how to maintain reliable communication as the link conditions are affected by variations in signal propagation due to the movement of the UAVs. Particularly, the antenna characteristics (radiation pattern and polarization) and orientation has strong impact on the link performance [3]. Second, these applications also require that the information should be delivered with low latency [4]. Third, power consumption is a limitation for UAV networks, as UAVs are powered by battery or fuel cells with limited life time (i.e. few minutes to few hours).

Currently, existing wireless technologies, such as Wireless Fidelity (WiFi) and ZigBee are being used for communication with the UAVs. However, their usage is limited to very short range, low throughput, and low-mobility applications. Particularly, these technologies are not suitable for applications where a swarm of UAVs needs simultaneous communication with the GS through a high-throughput link (e.g. 20-30 small UAVs transmitting high-resolution videos to the GS). The list of such civilian and military applications for UAVs keeps growing [2], [5]-[7]. Therefore, a new breakthrough technology is required in order to support the multitude of applications of UAVs that need high throughput, low power consumption, and low latency.

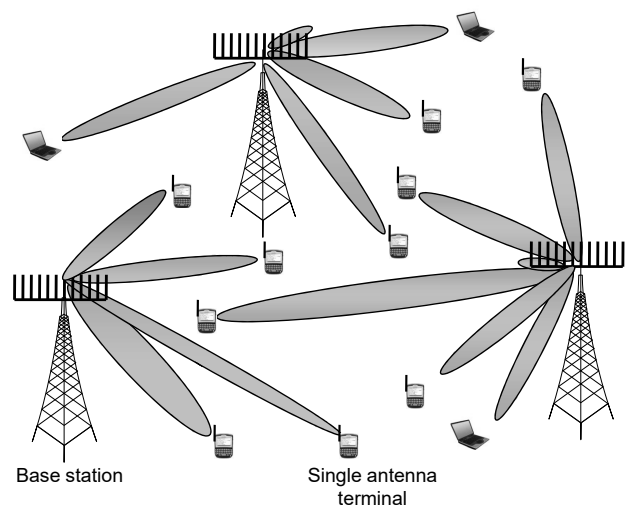

Fig. 1. Massive MIMO cellular system

Massive multiple input multiple output (MIMO) system is an emerging technique due to its scalability and potential to meet the high throughput requirements of the next generation cellular systems [8], [9]. In massive MIMO cellular system, base stations equipped with a very large numbers of antennas simultaneously serve multiple single-antenna terminals (see Fig. 1). By coherent closed-loop beamforming, the energy is focused into a small region of space and thus reducing interference. It also provides significant improvement in energy efficiency and reduced latency [10]. To avoid channel state information feedback, Massive MIMO uses timedivision multiplexing (TDD), exploiting channel reciprocity. In this paper, we study how Massive MIMO technology could facilitate high-performance UAV communication networks. 


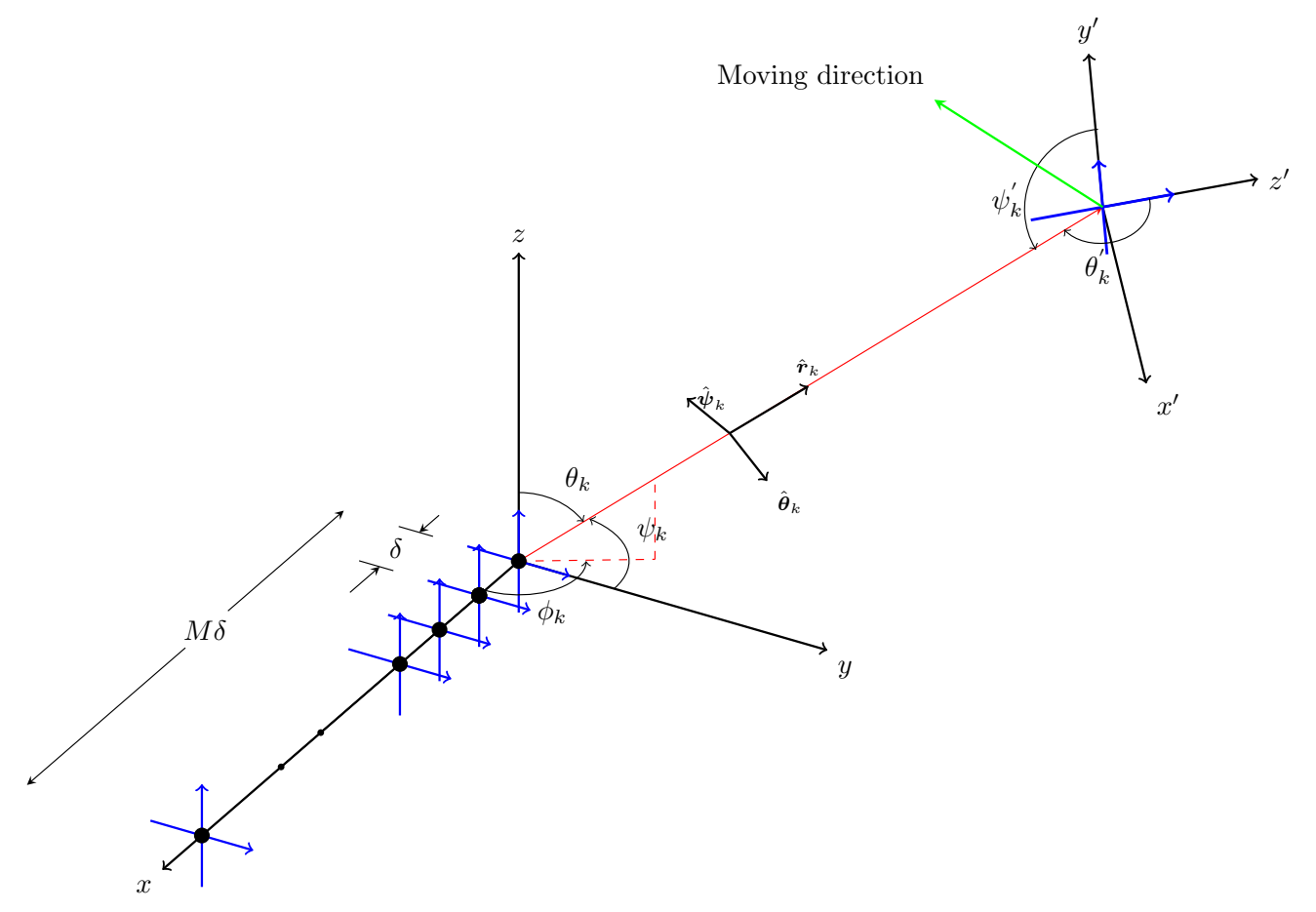

Fig. 2. Illustration of 3D geometric model

\section{A. Contributions}

As an initial study, we ask and answer the following questions:

- What is the achievable uplink capacity when a GS equipped with a large number of antennas simultaneously communicates with a swarm of single-antenna, rapidly moving UAVs?

- How large reduction in UAV transmit power is possible compared to a single-antenna GS?

- How does the antenna configuration (i.e. orientation and polarization) affect the link budget, and what is the appropriate antenna polarization that should be used in order to maintain a reliable communication link?

We consider a scenario where multiple single-antenna UAVs simultaneously communicate with a GS which is equipped with a uniform linear array (ULA). We quantify the achievable uplink capacity performance in the case of lineof-sight (LoS) conditions in terms of number of antennas. In order to make the analysis simpler, we assume that perfect channel state information (CSI) is available. Future work will analyze the achievable capacity performance with estimated CSI. Further, in surveillance, search and rescue operations, the rotation of UAVs while moving changes the orientation of the antenna as well, which results in a polarization mismatch at the receiver side. This can lead to poor link performance [3], [11]. Therefore, we develop a realistic geometric model which captures the polarization characteristics of the GS and UAV antennas. By using this model, we study the effect of employing different antenna polarization for achieving reliable communication link.

\section{B. Related Works}

There is some previous work that addresses the issue of communication with the UAVs. The challenges of existing wireless technologies, such as WiFi, ZigBee, and WiMAX for UAV communications in alpine environments were discussed in [12]. The use of IEEE 802.11n for UAV communications was experimentally studied in [3], [11]. However, since the coverage of $802.11 \mathrm{n}$ is limited to short range, it is not suitable for long ranges and high-mobility applications where the flying speed of the UAVs is in the range of $20-30 \mathrm{~m} / \mathrm{s}$ [3]. Further, the above mentioned works consider a scenario where communication takes place only between two nodes, i.e. either between the GS and a single UAV or between two UAVs. A simulation-based study on the utilization of multiuser MIMO communication for air traffic management for the airplanes flying at altitudes ranging from $5 \mathrm{~km}$ to 10 $\mathrm{km}$ was presented in [13]. The authors studied the impact of antenna spacing on the sum capacity performance in uplink. However, they have neither used a detailed geometric model nor studied the impact of the number of antennas on the achievable capacity. 


\section{SYSTEM MODEL}

\section{A. Geometric Model}

We consider the uplink of air to ground communication system. We consider an environment with only LoS propagation, since it is the most appropriate channel model for UAV communications. The geometric model of the system is illustrated in Fig. 2. For simplicity of the analysis, in this work we consider an ULA. In our future work, we plan to use a generalized array structure model with an optimized antenna spacing. We consider a Cartesian coordinate system with orthogonal unit directions $(\hat{\boldsymbol{x}}, \hat{\boldsymbol{y}}, \hat{\boldsymbol{z}})$ as a reference coordinate system. The array is located along the $x$-axis, and the first antenna being at the origin. Each antenna element is composed of two dipoles (one dipole is $z$ directed and an another dipole is $y$ directed). The spacing between the antenna elements is denoted by $\delta$. The $l$-th antenna position is denoted by $\left(x^{(l)}, y^{(l)}, z^{(l)}\right)=((l-1) \delta, 0,0), l \in\{1,2, \ldots, M\}$.

There are $K$ single-antenna UAVs simultaneously transmitting data in the same time-frequency resource to the GS. The UAV antenna is composed of two crossed dipoles. Note that the crossed dipoles can be used to obtain linear and circular polarized waves [14, Sec: 2.12.2]. The position of $k$-th UAV's antenna in spherical coordinate is denoted by the vector $\left(d_{k}, \theta_{k}, \phi_{k}\right)$, where $d_{k}$ is the radial distance between the GS and the UAV, $\phi_{k} \in[0,2 \pi]$ is the azimuth angle (i.e. the angle from the positive $x$-axis toward the positive $y$-axis, to the vector's orthogonal projection onto the $x$ $y$ plane), and $\theta_{k} \in[0, \pi]$ is the elevation angle (i.e. the angle from the positive $z$-axis toward the position vector). In Cartesian coordinates the $x, y$, and $z$ components are given by $\left(x_{k}, y_{k}, z_{k}\right)=\left(d_{k} \cos \phi_{k} \sin \theta_{k}, d_{k} \sin \phi_{k} \sin \theta_{k}, d_{k} \cos \theta_{k}\right)$. The distance between the $l$-th GS antenna and the $k$-th UAV's antenna is given by

$$
d_{k l}=\sqrt{\left(x_{k}-(l-1) \delta\right)^{2}+y_{k}^{2}+z_{k}^{2}} .
$$

By expanding (1), we get

$$
d_{k l}=d_{k}\left(1+\frac{1}{d_{k}^{2}}(l-1)^{2} \delta^{2}-\frac{2}{d_{k}}(l-1) \delta \sin \theta_{k} \cos \phi_{k}\right)^{\frac{1}{2}} .
$$

The azimuth angle of arrival of the incident wave on the $l$-th GS antenna is given by

$$
\phi_{k l}=\tan ^{-1}\left[\frac{d_{k} \sin \phi_{k} \sin \theta_{k}}{d_{k} \cos \phi_{k} \sin \theta_{k}-(l-1) \delta}\right] .
$$

The elevation angle of arrival of the incident wave on the $l$-th GS antenna is given by

$$
\theta_{k l}=\cos ^{-1}\left[\frac{d_{k} \cos \theta_{k}}{d_{k l}}\right] \text {. }
$$

\section{B. UAV Rotation Model}

The polarization mismatch effect due to change of antenna orientation is generally incorporated using a rotational matrix [15]-[17]. We use a similar approach to model the polarization characteristics of GS and UAV antennas.

The 3-D rotation is easier to express in Cartesian coordinates. Therefore, we describe the rotation of UAVs using Cartesian coordinates. The UAV's rotation around the coordinate axes is denoted by 1) Roll $\left(\alpha_{x}\right)$ : angle of rotation around $x$-axis 2) Pitch $\left(\alpha_{y}\right)$ : angle of rotation around $y$-axis 3) Yaw $\left(\alpha_{z}\right)$ : angle of rotation around $z$-axis. For example, Fig. 3 shows the transformed unit direction vectors due to a rotation around the $x$-axis i.e.

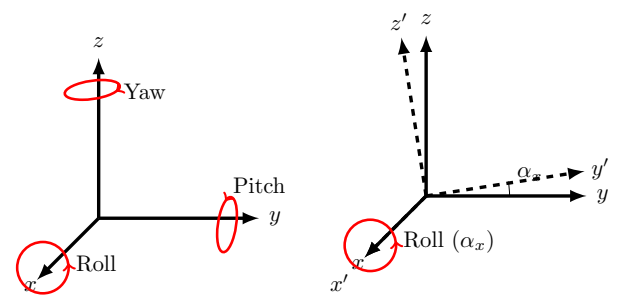

Fig. 3. UAV rotation model

$$
\left[\begin{array}{l}
\hat{\boldsymbol{x}}^{\prime} \\
\hat{\boldsymbol{y}}^{\prime} \\
\hat{\boldsymbol{z}}^{\prime}
\end{array}\right]=\left[\begin{array}{ccc}
1 & 0 & 0 \\
0 & \cos \alpha_{x} & \sin \alpha_{x} \\
0 & -\sin \alpha_{x} & \cos \alpha_{x}
\end{array}\right]\left[\begin{array}{l}
\hat{\boldsymbol{x}} \\
\hat{\boldsymbol{y}} \\
\hat{\boldsymbol{z}}
\end{array}\right] .
$$

The $3 \times 3$ matrix in (5) is denoted by $\mathbf{R}_{3, x}\left(\alpha_{x}\right)$. Similarly, the rotation matrices around $y$ and $z$ axes are denoted by $\mathbf{R}_{3, y}\left(\alpha_{y}\right)$ and $\mathbf{R}_{3, z}\left(\alpha_{z}\right)$, respectively. This rotation matrix is used to calculate the resultant channel gain between an GS antenna and the UAV as detailed in the next section. In practice, the rotation of the UAV may take place at around any of the three axes at any time irrespective of the current state of the rotation. In that case, the elements of the rotation matrix will be a function of roll, pitch, and yaw angles $\alpha_{x}, \alpha_{y}$, and $\alpha_{z}$, respectively. Further, the elements in the rotation matrix depend on the order that the axes are rotated. For example, the rotation matrix of the $k$-th UAV that rotates in the order around $z, y$, and $x$ axes is obtained by $\mathbf{R}_{3}^{(k)}\left(\alpha_{z}, \alpha_{y}, \alpha_{x}\right)=\mathbf{R}_{3, x}^{(k)}\left(\alpha_{x}\right) \mathbf{R}_{3, y}^{(k)}\left(\alpha_{y}\right) \mathbf{R}_{3, z}^{(k)}\left(\alpha_{z}\right)$. Let

$$
\mathbf{R}_{3}^{(k)}\left(\alpha_{z}, \alpha_{y}, \alpha_{x}\right)=\left[\begin{array}{ccc}
\mathbf{R}_{11}^{(k)} & \mathbf{R}_{12}^{(k)} & \mathbf{R}_{13}^{(k)} \\
\mathbf{R}_{21}^{(k)} & \mathbf{R}_{22}^{(k)} & \mathbf{R}_{23}^{(k)} \\
\mathbf{R}_{31}^{(k)} & \mathbf{R}_{32}^{(k)} & \mathbf{R}_{33}^{(k)}
\end{array}\right] .
$$

Then the transformed Cartesian coordinate axes due to rotation of the $k$-th UAV UAV can be written as

$$
\begin{aligned}
\hat{\boldsymbol{x}}_{k}^{\prime} & =\mathbf{R}_{11}^{(k)} \hat{\boldsymbol{x}}+\mathbf{R}_{12}^{(k)} \hat{\boldsymbol{y}}+\mathbf{R}_{13}^{(k)} \hat{\boldsymbol{z}} \\
\hat{\boldsymbol{y}}_{k}^{\prime} & =\mathbf{R}_{21}^{(k)} \hat{\boldsymbol{x}}+\mathbf{R}_{22}^{(k)} \hat{\boldsymbol{y}}+\mathbf{R}_{23}^{(k)} \hat{\boldsymbol{z}} \\
\hat{\boldsymbol{z}}_{k}^{\prime} & =\mathbf{R}_{31}^{(k)} \hat{\boldsymbol{x}}+\mathbf{R}_{32}^{(k)} \hat{\boldsymbol{y}}+\mathbf{R}_{33}^{(k)} \hat{\boldsymbol{z}} .
\end{aligned}
$$




\section{Polarization Model}

Polarization is usually described by the orientation of electric field vector over time at a constant point. The orientation of electric field is always orthogonal to the direction of wave propagation. For example, for a dipole antenna placed along the $z$ axis (see Fig. 2), if the wave travels in the $\hat{\boldsymbol{r}}_{k}$ direction, the electric field orientation will be in the $\hat{\boldsymbol{\theta}}_{k}$ direction and the magnetic field orientation will be in the $\hat{\phi}_{k}$ direction. The unit direction vectors in spherical coordinates with respect to the reference coordinate system is given by

$$
\begin{aligned}
& \hat{\boldsymbol{r}}_{k}=\sin \theta_{k} \cos \phi_{k} \hat{\boldsymbol{x}}+\sin \theta_{k} \sin \phi_{k} \hat{\boldsymbol{y}}+\cos \theta_{k} \hat{\boldsymbol{z}}, \\
& \hat{\boldsymbol{\theta}}_{k}=\cos \theta_{k} \cos \phi_{k} \hat{\boldsymbol{x}}+\cos \theta_{k} \sin \phi_{k} \hat{\boldsymbol{y}}-\sin \theta_{k} \hat{\boldsymbol{z}}, \\
& \hat{\boldsymbol{\phi}}_{k}=-\sin \phi_{k} \hat{\boldsymbol{x}}+\cos \phi_{k} \hat{\boldsymbol{y}} .
\end{aligned}
$$

For the dipole antenna placed parallel to the $y$ axis, the elevation angle $\psi_{k}$ can be obtained from the scalar product between the unit direction vectors $\hat{\boldsymbol{y}}$ and $\hat{\boldsymbol{r}}_{k}$ as

$$
\psi_{k}=\cos ^{-1}\left(\hat{\boldsymbol{y}} \cdot \hat{\boldsymbol{r}}_{k}\right)=\cos ^{-1}\left(\sin \theta_{k} \sin \phi_{k}\right) .
$$

Taking into consideration the fact that the unit vector $\hat{\boldsymbol{\psi}}_{k}$ is orthogonal to $\hat{\boldsymbol{r}}_{k}$ and parallel to the plane generated by the vectors $\hat{\boldsymbol{r}}_{k}$ and $\hat{\boldsymbol{y}}$, we obtain

$$
\hat{\boldsymbol{\psi}}_{k}=\cot \psi_{k} \sin \theta_{k} \cos \phi_{k} \hat{\boldsymbol{x}}-\sin \psi_{k} \hat{\boldsymbol{y}}+\cot \psi_{k} \cos \theta_{k} \hat{\boldsymbol{z}} \text {. }
$$

Next we calculate the elevation angles from the $k$-th UAV antenna. Let $\hat{\boldsymbol{r}}_{k}^{\prime}$ be the wave travel direction measured from the $k$-th UAV antenna i.e.

$$
\hat{\boldsymbol{r}}_{k}^{\prime}=-\hat{\boldsymbol{r}}_{k}
$$

Then the elevation angles from the $k$-th UAV antennas oriented along the $\hat{\boldsymbol{z}}_{k}^{\prime}$ and $\hat{\boldsymbol{y}}_{k}^{\prime}$ directions are obtained as follows. Since $\cos \theta_{k}^{\prime}=\hat{\boldsymbol{z}}_{k}^{\prime} \cdot \hat{\boldsymbol{r}}_{k}^{\prime}$, for the dipole antenna oriented along the $\hat{\boldsymbol{z}}_{k}^{\prime}$ direction, the elevation angle is obtained as

$$
\begin{aligned}
& \theta_{k}^{\prime}=\cos ^{-1}\left(\hat{\boldsymbol{z}}_{k}^{\prime} \cdot \hat{\boldsymbol{r}}_{k}^{\prime}\right) \\
& =\cos ^{-1}\left(-\sin \theta_{k} \cos \phi_{k} \mathbf{R}_{31}^{(k)}-\sin \theta_{k} \sin \phi_{k} \mathbf{R}_{32}^{(k)}-\cos \theta_{k} \mathbf{R}_{33}^{(k)}\right) .
\end{aligned}
$$

Similarly, since $\cos \psi_{k}^{\prime}=\hat{\boldsymbol{y}}_{k}^{\prime} \cdot \hat{\boldsymbol{r}}_{k}^{\prime}$, for the dipole antenna oriented along the $\hat{\boldsymbol{y}}_{k}^{\prime}$ direction, the elevation angle is obtained as

$$
\begin{aligned}
& \psi_{k}^{\prime}=\cos ^{-1}\left(\hat{\boldsymbol{y}}_{k}^{\prime} \cdot \hat{\boldsymbol{r}}_{k}^{\prime}\right) \\
& =\cos ^{-1}\left(-\sin \theta_{k} \cos \phi_{k} \mathbf{R}_{21}^{(k)}-\sin \theta_{k} \sin \phi_{k} \mathbf{R}_{22}^{(k)}-\cos \theta_{k} \mathbf{R}_{23}^{(k)}\right) .
\end{aligned}
$$

Next we calculate the channel gain from the GS antenna to the UAV antenna. Due to reciprocity, this is same as the channel gain from the UAV antenna to the GS antenna.
Let $E_{G S}^{\theta}$ and $E_{G S}^{\psi}$ denote the complex magnitude of field components transmitted by the GS dipoles oriented along the $z$-axis and $y$-axis, respectively. Note that the polarization (i.e. linear or circular or elliptical) of the resultant wave is determined by the magnitudes and time-phase difference between the quantities $E_{G S}^{\theta}$ and $E_{G S}^{\psi}$. For more details see [14, Sec: 2.12]. Let $\cos a_{1}$ and $\cos b_{1}$ be the magnitudes of the projection of the unit vectors $\hat{\boldsymbol{\theta}}_{k}$ and $\hat{\boldsymbol{\psi}}_{k}$, respectively, on to the dipole antenna of the $k$-th UAV oriented along the $\hat{z}_{k}^{\prime}$-axis i.e.

$$
\begin{aligned}
\cos a_{1} & =\hat{\boldsymbol{\theta}}_{k} . \hat{\boldsymbol{z}}_{k}^{\prime} \\
& =\cos \theta_{k} \cos \phi_{k} \mathbf{R}_{31}^{(k)}+\cos \theta_{k} \sin \phi_{k} \mathbf{R}_{32}^{(k)}-\sin \theta_{k} \mathbf{R}_{33}^{(k)} \\
\cos b_{1} & =\hat{\boldsymbol{\psi}}_{k} \cdot \hat{\boldsymbol{z}}_{k}^{\prime} \\
& =\cot \psi_{k} \sin \theta_{k} \cos \phi_{k} \mathbf{R}_{31}^{(k)}-\sin \psi_{k} \mathbf{R}_{32}^{(k)} \\
& \quad+\cot \psi_{k} \cos \theta_{k} \mathbf{R}_{33}^{(k)} .
\end{aligned}
$$

Now the total electric filed received by the $k$-th UAV antenna oriented along the $\hat{\boldsymbol{z}}_{k}^{\prime}$-axis is the sum of the field components projected from the electric field vectors in $\hat{\boldsymbol{\theta}}_{k}$ and $\hat{\psi}_{k}$ directions i.e.

$$
\begin{aligned}
\mathrm{E}_{U A V}^{\theta}= & E_{G S}^{\theta} F_{G S}^{\theta}\left(\theta_{k}\right) F_{U A V}^{\theta}\left(\theta_{k}^{\prime}\right) \cos a_{1} \\
& +E_{G S}^{\psi} F_{G S}^{\psi}\left(\psi_{k}\right) F_{U A V}^{\theta}\left(\theta_{k}^{\prime}\right) \cos b_{1},
\end{aligned}
$$

where $F_{G S}^{\theta}\left(\theta_{k}\right)$ and $F_{G S}^{\psi}\left(\psi_{k}\right)$ are the antenna gain patterns of $z$ directed and $y$ directed dipole antennas, respectively.

Similarly, let $\cos a_{2}$ and $\cos b_{2}$ be the magnitudes of the projection of the vectors $\hat{\boldsymbol{\theta}}_{k}$ and $\hat{\boldsymbol{\psi}}_{k}$, respectively, on to the dipole antenna of the $k$-th UAV oriented along the $\hat{\boldsymbol{y}}^{\prime}$-axis i.e.

$$
\begin{aligned}
\cos a_{2} & =\hat{\boldsymbol{\theta}}_{k} \cdot \hat{\boldsymbol{y}}_{k}^{\prime} \\
& =\cos \theta_{k} \cos \phi_{k} \mathbf{R}_{21}^{(k)}+\cos \theta_{k} \sin \phi_{k} \mathbf{R}_{22}^{(k)}-\sin \theta_{k} \mathbf{R}_{23}^{(k)} \\
\cos b_{2} & =\hat{\boldsymbol{\psi}}_{k} \cdot \hat{\boldsymbol{y}}_{k}^{\prime} \\
& =\cot \psi_{k} \sin \theta_{k} \cos \phi_{k} \mathbf{R}_{21}^{(k)}-\sin \psi_{k} \mathbf{R}_{22}^{(k)} \\
& \quad+\cos \psi_{k} \cot \theta_{k} \mathbf{R}_{23}^{(k)} .
\end{aligned}
$$

The total electric filed received by the dipole antenna of the $k$-th UAV oriented along the $\hat{\boldsymbol{y}}_{k}^{\prime}$-axis is

$$
\begin{aligned}
\mathrm{E}_{U A V}^{\psi}= & E_{G S}^{\theta} F_{G S}^{\theta}\left(\theta_{k}\right) F_{U A V}^{\psi}\left(\psi_{k}^{\prime}\right) \cos a_{2} \\
& +E_{G S}^{\psi} F_{G S}^{\psi}\left(\psi_{k}\right) F_{U A V}^{\psi}\left(\psi_{k}^{\prime}\right) \cos b_{2} .
\end{aligned}
$$

Thus, the total electric field received by the $k$-th UAV antenna is obtained as the sum of the fields obtained from both the $\hat{\boldsymbol{z}}_{k}^{\prime}$ and $\hat{\boldsymbol{y}}_{k}^{\prime}$ directed antennas i.e.

$$
\mathrm{E}_{U A V}=\mathrm{E}_{U A V}^{\theta}+\mathrm{E}_{U A V}^{\psi} .
$$




\section{Channel Model}

The channel gain between the $k$-th UAV antenna and the $l$ th GS antenna is characterized by the following components: distance dependent pathloss, antenna patterns, polarization loss factor, and the phase factor.

Let the response vectors be

$$
\begin{array}{cc}
\mathbf{E}_{G S}\left(\theta_{k l}, \psi_{k l}\right)= & {\left[\begin{array}{l}
E_{G S}^{\theta} F_{G S}^{\theta}\left(\theta_{k l}\right) \\
E_{G S}^{\psi} F_{G S}^{\psi}\left(\psi_{k l}\right)
\end{array}\right] \text { and }} \\
\mathbf{E}_{U A V}\left(\theta_{k l}^{\prime}, \psi_{k l}^{\prime}\right)= & {\left[\begin{array}{l}
E_{U A V}^{\theta} F_{U A V}^{\theta}\left(\theta_{k l}^{\prime}\right) \\
E_{U A V}^{\psi} F_{U A V}^{\psi}\left(\psi_{k l}^{\prime}\right)
\end{array}\right] .}
\end{array}
$$

Let the $2 \times 2$ matrix that represents the polarization mismatch be

$$
\mathbf{T}_{2}=\left[\begin{array}{ll}
\cos a_{1} & \cos a_{2} \\
\cos b_{1} & \cos b_{2}
\end{array}\right] .
$$

The effective gain (including polarization mismatch factors) between the $k$-th UAV antenna and the $l$-th GS antenna can be written as

$$
E_{k l}=\mathbf{E}_{G S}\left(\theta_{k l}, \psi_{k l}\right)^{T} \mathbf{T}_{2} \mathbf{E}_{U A V}\left(\theta_{k l}^{\prime}, \psi_{k l}^{\prime}\right) .
$$

The polarization loss factor (PLF) between the $l$-th GS antenna and the $k$-th UAV antenna is defined as

$$
P L F_{k l}=\frac{\left|E_{k l}\right|^{2}}{\left|\mathbf{E}_{G S}\left(\theta_{k l}, \psi_{k l}\right)\right|^{2}\left|\mathbf{E}_{U A V}\left(\theta_{k l}^{\prime}, \psi_{k l}^{\prime}\right)\right|^{2}} .
$$

Note that in case of single $z$ directed dipole antenna both at the GS and the UAV (i.e. $E_{G S}^{\theta}=E_{U A V}^{\theta}=1$ and $E_{G S}^{\psi}=$ $E_{U A V}^{\psi}=0$ ), the PLF becomes $P L F_{k l}=\left|\cos a_{1}\right|^{2}$.

Finally, the $M \times 1$ channel vector from the $k$-th UAV to the GS is given by

$$
\boldsymbol{g}_{k}=\left[\begin{array}{llll}
g_{k 1} & g_{k 2} & \ldots & g_{k M}
\end{array}\right]^{T},
$$

where $g_{k l}, l=1,2, \ldots, M$ denotes the complex channel coefficients between the $l$-th GS antenna and the UAV i.e.

$$
g_{k l}=\sqrt{\beta_{k l}} E_{k l} e^{-i \frac{2 \pi}{\lambda} d_{k l}},
$$

where $\lambda$ is the carrier wavelength and $\beta_{k l}$ is distance dependent pathloss component.

\section{E. Uplink Data Transmission}

Let the $M \times K$ channel matrix between the GS and the $\mathrm{UAV}$ s as

$$
\boldsymbol{G}=\left[\boldsymbol{g}_{1}, \ldots, \boldsymbol{g}_{K}\right] .
$$

The $M \times 1$ received signal vector at the GS is given by

$$
\boldsymbol{y}=\sqrt{p_{u}} \boldsymbol{G} \boldsymbol{x}+\boldsymbol{n},
$$

where $\sqrt{p_{u}} \boldsymbol{x}$ is the vector of symbols simultaneously transmitted by the $K$ UAVs i.e. $\sqrt{p_{u}} \boldsymbol{x}=\sqrt{p_{u}}\left[x_{1}, x_{2}, \ldots, x_{K}\right]^{T}$ (normalized such that $\mathbb{E}\left\{\left|x_{k}\right|^{2}\right\}=1$ for all $k \in$ $\{1,2, \ldots, K\}) ; \boldsymbol{n}$ is a complex AWGN vector, $\boldsymbol{n} \sim$
$\mathcal{C N}\left(0, N_{0} \boldsymbol{I}_{M}\right)$, where $\boldsymbol{I}_{M}$ is an identity matrix of size $M \times M$ and $N_{0}$ is noise power spectral density in $\mathrm{W} / \mathrm{Hz}$.

Next we compute the post-processing SINR at the output of the maximum ratio combining (MRC) detector by assuming that perfect CSI is available at the GS. With perfect CSI, the post-processing SINR of $k$-th UAV at the output of the MRC receiver is given by [10]

$$
\gamma_{k}=\frac{p_{u}\left\|\boldsymbol{g}_{k}\right\|^{4}}{p_{u} \sum_{j=1, j \neq k}^{K}\left|\boldsymbol{g}_{k}^{H} \boldsymbol{g}_{j}\right|^{2}+\left\|\boldsymbol{g}_{k}\right\|^{2} N_{0}} .
$$

From (24) and (25),

$$
\left\|\boldsymbol{g}_{k}\right\|=\sqrt{\sum_{l=1}^{M} \beta_{k l} \chi_{k l}},
$$

where $\chi_{k l}=\left|E_{k l}\right|^{2}$. The square of inner product between the spatial signatures of the $k$-th and the $j$-th UAVs is given by

$$
\left|\boldsymbol{g}_{k}^{H} \boldsymbol{g}_{j}\right|^{2}=\left|\sum_{l=1}^{M} \sqrt{\beta_{k l} \beta_{j l} \chi_{k l} \chi_{j l}} e^{i \frac{2 \pi}{\lambda}\left(d_{k l}-d_{j l}\right)}\right|^{2} .
$$

Finally, by substituting (29) and (30) into (28), the instantaneous uplink SINR of $k$-th UAV is obtained as

$$
=\frac{\left[\sum_{l=1}^{M} \beta_{k l} \chi_{k l}\right]^{2}}{\sum_{j=1, j \neq k}^{K}\left|\sum_{l=1}^{M} \sqrt{\beta_{k l} \beta_{j l} \chi_{k l} \chi_{j l}} e^{i \frac{2 \pi}{\lambda}\left(d_{k l}-d_{j l}\right)}\right|^{2}+\frac{N_{0}}{p_{u}} \sum_{l=1}^{M} \beta_{k l} \chi_{k l}} .
$$

\section{CAPACITy AnAlysis}

In this section, we discuss the achievable capacity performance for UAV communications. For simplicity of analysis, we consider a scenario where the distances between the GS and the UAVs are larger than the array size i.e. $M \delta<<$ $d_{k}, \forall k$. By using the approximation $\sqrt{1+x} \approx 1+\frac{x}{2}$, the distance $d_{k l}$ in (2) is simplified to

$$
d_{k l} \approx d_{k}-(l-1) \delta \sin \theta_{k} \cos \phi_{k} .
$$

With this assumption, from (3) and (4) for all $l \in$ $\{1,2, \ldots, M\}$, we get

$$
\begin{aligned}
& \theta_{k l} \approx \theta_{k}, \phi_{k l} \approx \phi_{k}, \theta_{k l}^{\prime} \approx \theta_{k}^{\prime}, \\
& \phi_{k l}^{\prime} \approx \phi_{k}^{\prime}, \beta_{k l} \approx \beta_{k}, \chi_{k l} \approx \chi_{k} .
\end{aligned}
$$

By applying (32) and (33), the numerator term in (31) is constantly equal to $p_{u} \beta_{k}^{2} \chi_{k}^{2} M^{2}$. Therefore, the analysis of SINR depends on the quantity $\left|\boldsymbol{g}_{k}^{H} \boldsymbol{g}_{j}\right|^{2}$. By applying (33), (30) is simplified to

$$
\begin{aligned}
\left|\boldsymbol{g}_{k}^{H} \boldsymbol{g}_{j}\right|^{2}= & \beta_{k} \beta_{j} \chi_{k} \chi_{j} \\
& \times\left|\sum_{n=0}^{M-1} e^{i \frac{2 \pi}{\lambda} n \delta\left(\sin \theta_{j} \cos \phi_{j}-\sin \theta_{k} \cos \phi_{k}\right)}\right|^{2} .
\end{aligned}
$$




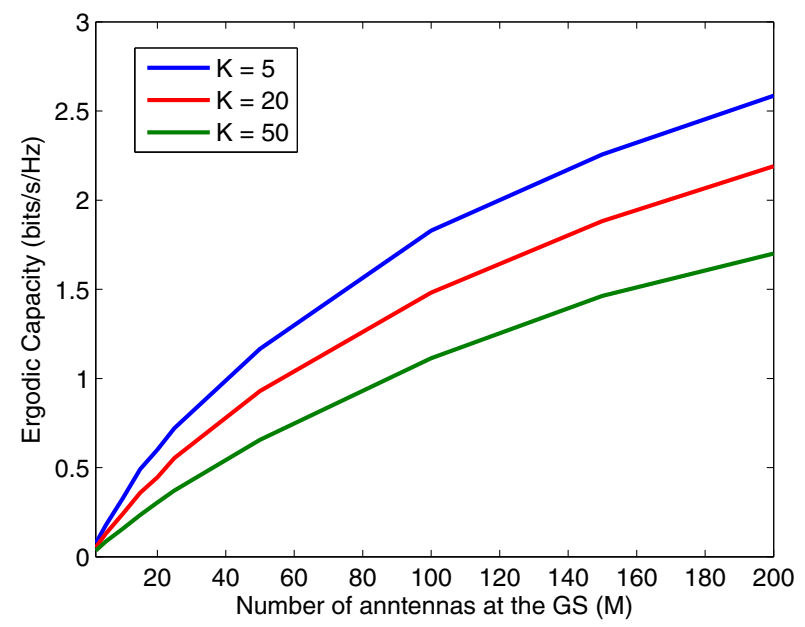

Fig. 4. Lower bound of ergodic capacity for different numbers of GS antennas for MRC with perfect CSI. In this example, the signal to noise ratio is $\frac{p_{u}}{N_{0}}=30 \mathrm{~dB}$.

The quantity $\left|\boldsymbol{g}_{k}^{H} \boldsymbol{g}_{j}\right|^{2}$ will be continuously changing, as the elevation and azimuth angles between the UAVs and the GS are changing due to the movement of the UAVs. In practice, since multiple UAVs will coexist in the environment, it is more likely that any of the UAVs will interfere the intended UAV. Further, one can expect multiple realizations of the quantity $\left|\boldsymbol{g}_{k}^{H} \boldsymbol{g}_{j}\right|^{2}$ within the codeword transmission time. Therefore, next we compute a lower bound of ergodic capacity by averaging over all possible realizations of the quantity $\left|\boldsymbol{g}_{k}^{H} \boldsymbol{g}_{j}\right|^{2}$.

\section{A. Ergodic Capacity}

The instantaneous uplink capacity achieved by the $k$-th UAV is given by $R_{k}=\log _{2}\left(1+\gamma_{k}\right)$. Then, the ergodic capacity is

$$
\bar{R}_{k}=\mathbb{E}\left\{R_{k}\right\}=\mathbb{E}\left\{\log _{2}\left(1+\gamma_{k}\right)\right\} \text { bits/s/Hz. }
$$

By the convexity of $\log _{2}\left(1+\frac{1}{z}\right)$ (for $z \geq 0$ ) and using Jensen's inequality $(\mathbb{E}\{f(z)\} \geq f(\mathbb{E}(z))$, where $f(z)$ is a convex function), the ergodic capacity is lower bounded by

$$
\bar{R}_{k} \geq \bar{R}_{k}^{l b} \triangleq \log _{2}\left(1+\frac{1}{\mathbb{E}\left\{\frac{1}{\gamma_{k}}\right\}}\right) .
$$

With MRC, under the assumption of perfect CSI, we get the lower bound of ergodic capacity (in bits/s/Hz)

$$
\bar{R}_{k}^{l b}=\log _{2}\left(1+\frac{\beta_{k} \chi_{k} M}{\left(1+\frac{\Omega}{M}\right) \sum_{j=1, j \neq k}^{K} \beta_{j} \chi_{j}+\frac{N_{0}}{p_{u}}}\right),
$$

where

$$
\Omega=\sum_{m=0}^{M-1} \sum_{n=0, n \neq m}^{M-1} \operatorname{sinc}^{2}(2(m-n) \delta / \lambda) .
$$

For proof of (37), see Appendix A.

\section{RESUlts}

\section{A. Achievable ergodic capacity}

In this section, we present the ergodic capacity results to show the potential of using massive MIMO for UAV communications. The UAVs are assumed to be uniformly distributed within a spherical volume with radius $500 \mathrm{~m}$ and we assume that no UAV is close to the GS than $10 \mathrm{~m}$. Further, we choose carrier frequency $f=2.4 \mathrm{GHz}$, antenna spacing $\delta=0.5 \lambda=6.25 \mathrm{~cm}$, system bandwidth $B=10 \mathrm{MHz}$, $N_{0}=k_{B} T 10^{\mathcal{F} / 10} \approx 2 \times 10^{-20} \mathrm{~J}$, where $k_{B}=1.38 \times 10^{-23}$ $\mathrm{J} / \mathrm{K}, \mathrm{T}=300 \mathrm{~K}$, and the receiver noise figure $\mathcal{F}=7 \mathrm{~dB}$. The received signal power at each GS antenna is calculated by

$$
\begin{aligned}
P_{r}(d B m)=p_{u} & (d B m)+20 \log _{10}\left(F_{G S}(\theta, \psi)\right) \\
& +20 \log _{10}\left(F_{U A V}\left(\theta^{\prime}, \psi^{\prime}\right)\right)-10 \log _{10}(P L F) \\
& +20 \log _{10}\left(\frac{\lambda}{4 \pi d}\right) .
\end{aligned}
$$

Fig. 4 shows the lower bound on ergodic capacity (with perfect CSI) as a function of number of antennas for different number of UAVs for $\frac{p_{u}}{N_{0}}=30 \mathrm{~dB}$ i.e. $p_{u}=2 \times 10^{-13} \mathrm{~W}$. In order to show the typical behavior of the ergodic capacity, we neglected the polarization mismatch loss. It can be seen that by employing large number of antennas at the GS, the uplink capacity is increased without adding extra power at the UAV. Note that in single antenna system, for an UAV, the average power required in order to achieve the target capacity of 1 $\mathrm{bits} / \mathrm{s} / \mathrm{Hz}$ is approximately $1.77 \times 10^{-8} \mathrm{~W}$ (this number is obtained by averaging over all positions of the UAV within a spherical volume of inner radius: $50 \mathrm{~m}$ and outer radius: $500 \mathrm{~m}$ ). Whereas, with massive MIMO, considering perfect CSI, by employing 40 antennas at the GS, the same target 


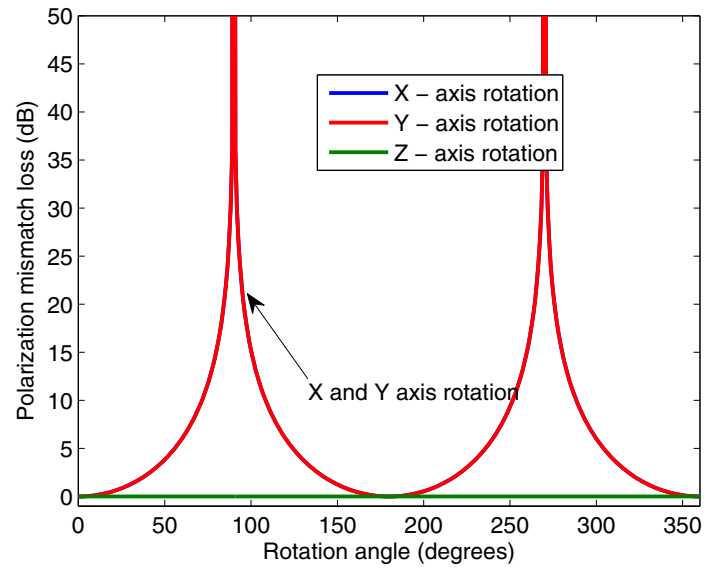

(a)

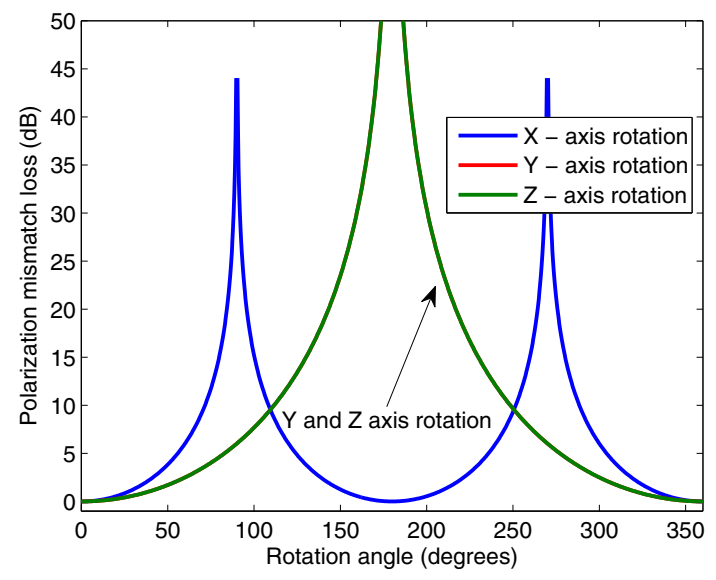

(c)

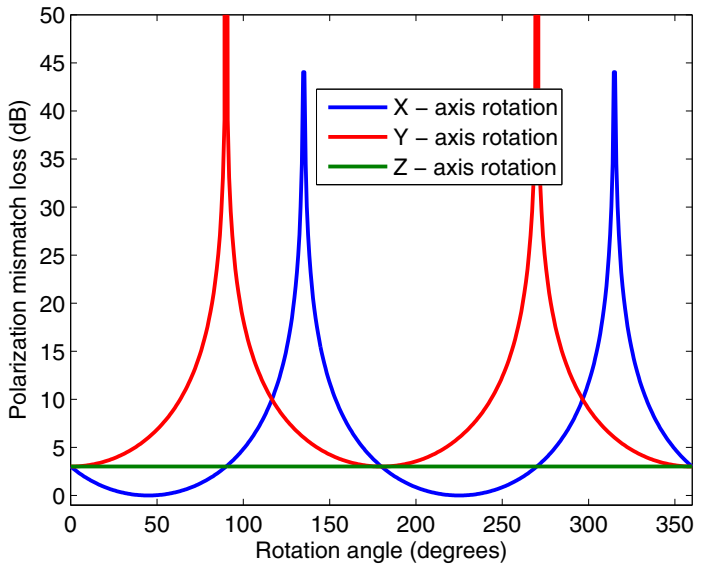

(b)

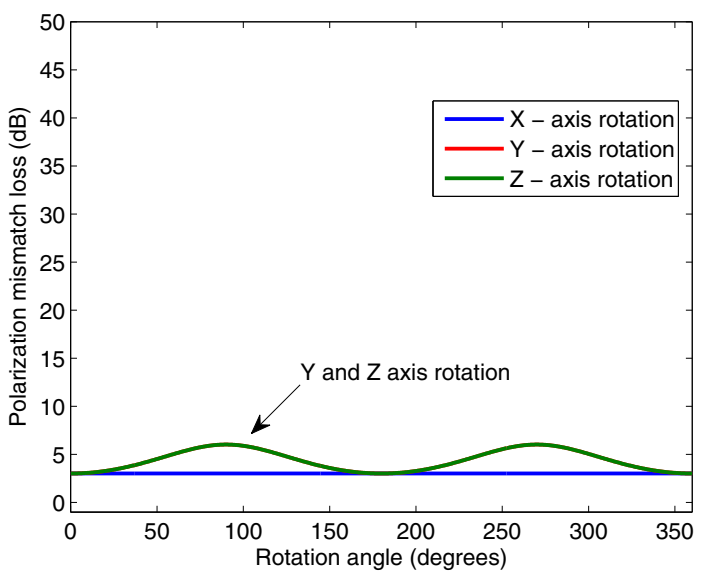

(d)

Fig. 5. Polarization loss due to rotation of the UAVs (a) $E_{G S}^{\theta}=1, E_{G S}^{\psi}=0, E_{U A V}^{\theta}=1, E_{U A V}^{\psi}=0$. (b) $E_{G S}^{\theta}=\frac{1}{\sqrt{2}}, E_{G S}^{\psi}=\frac{1}{\sqrt{2}}, E_{U A V}^{\theta}=1$, $E_{U A V}^{\psi}=0$. (c) $E_{G S}^{\theta}=\frac{1}{\sqrt{2}}, E_{G S}^{\psi}=\frac{1}{\sqrt{2}}, E_{U A V}^{\theta}=\frac{1}{\sqrt{2}}, E_{U A V}^{\psi}=\frac{1}{\sqrt{2}}$. (d) $E_{G S}^{\theta}=\frac{1}{\sqrt{2}}, E_{G S}^{\psi}=\frac{1}{\sqrt{2}} i, E_{U A V}^{\theta}=\frac{1}{\sqrt{2}}, E_{U A V}^{\psi}=\frac{1}{\sqrt{2}}$.

capacity can be simultaneously achieved by five UAVs with approximately $20 \mathrm{~dB}$ less power when compared to single antenna system.

Further, the number of UAVs that can be supported also increases with the number of antennas. For example, with a target capacity of $1 \mathrm{bit} / \mathrm{s} / \mathrm{Hz}$, by increasing the number of antennas from 40 to 85 , the number of supportable UAVs can be increased from 5 to 50 .

\section{B. Impact of $U A V$ rotation}

In this section, we show the impact of UAV rotation on the PLF (the fourth term in (38)) assuming that $\theta_{k}=\frac{\pi}{2}$ and $\phi_{k}=\pi$.

Fig. 5 shows the polarization mismatch loss due to the rotation of UAV for different combinations of polarization at the GS and the UAV. From Fig. 5(a), 5(b), and 5(c), it can be observed that in case of linearly polarized antennas at the GS or at the UAV the rotation around any one axis results in very high polarization loss at certain rotation angles. Whereas, minimal polarization mismatch loss can be achieved by employing circular polarization at the GS and the dual polarization at the UAV (see Fig. 5(d)). Therefore, it can be concluded that it is beneficial to use circular polarized antennas for UAV communications in order to avoid the polarization mismatch loss that occurs due to the rotation of UAVs.

\section{CONCLUSions}

We investigated the potential benefits of using massive MIMO for UAV communications. It is shown that by increasing the number of antennas at the GS, the uplink capacity of UAVs can be increased several folds when compared 
to single antenna system while without increasing UAV's transmit power. It is also seen that using circular polarized antennas either at the GS or the UAV would be beneficial to maintain better link conditions.

\section{APPENDIX A}

PRoOF OF EQUATION (37)

With MRC, under the assumption of perfect CSI, from (31)

$\mathbb{E}\left\{\frac{1}{\gamma_{k}}\right\}=\frac{p_{u} \sum_{j=1, j \neq k}^{K} \mathbb{E}\left\{\left|\boldsymbol{g}_{k}^{H} \boldsymbol{g}_{j}\right|^{2}\right\}+\beta_{k} \chi_{k} M N_{0}}{p_{u} \beta_{k}^{2} \chi_{k}^{2} M^{2}}$

From (34), we can write

$$
\begin{aligned}
& \mathbb{E}\left\{\left|\boldsymbol{g}_{k}^{H} \boldsymbol{g}_{j}\right|^{2}\right\} \\
& =\beta_{k} \beta_{j} \chi_{k} \chi_{j} \sum_{m=0}^{M-1} \sum_{n=0}^{M-1} \mathbb{E}\left\{e^{i 2 \pi \frac{m-n}{\lambda} \delta\left(\sin \theta_{k} \cos \phi_{k}-\sin \theta_{j} \cos \phi_{j}\right)}\right\} .
\end{aligned}
$$

If we assume that the UAV positions are independently and uniformly distributed within a spherical volume, the distributions of elevation and azimuth angle are given by

$$
f_{\theta_{k}}(\theta)=\frac{\sin \theta}{2}, 0 \leq \theta \leq \pi
$$

and

$$
f_{\phi_{k}}(\phi)=\frac{1}{2 \pi}, 0 \leq \phi \leq 2 \pi
$$

respectively.

Therefore, the expectation in (40) can be expressed as

$$
\begin{aligned}
\mathbb{E} & \left\{e^{i 2 \pi \frac{m-n}{\lambda} \delta\left(\sin \theta_{j} \cos \phi_{j}-\sin \theta_{k} \cos \phi_{k}\right)}\right\} \\
= & \int_{0}^{2 \pi} \int_{0}^{\pi} e^{i 2 \pi \frac{\delta}{\lambda}(m-n) \sin \theta \cos \phi} f_{\theta_{j}}(\theta) f_{\phi_{j}}(\phi) d \theta d \phi \\
& \times \int_{0}^{2 \pi} \int_{0}^{\pi} e^{i 2 \pi \frac{\delta}{\lambda}(n-m) \sin \theta \cos \phi} f_{\theta_{k}}(\theta) f_{\phi_{k}}(\phi) d \theta d \phi \\
=\frac{1}{4 \pi} & \int_{0}^{2 \pi} \int_{0}^{\pi} e^{i 2 \pi \frac{\delta}{\lambda}(m-n) \sin \theta \cos \phi} \sin \theta d \theta d \phi \\
& \times \frac{1}{4 \pi} \int_{0}^{2 \pi} \int_{0}^{\pi} e^{i 2 \pi \frac{\delta}{\lambda}(n-m) \sin \theta \cos \phi} \sin \theta d \theta d \phi .
\end{aligned}
$$

It can be shown that

$$
\frac{1}{4 \pi} \int_{0}^{2 \pi} \int_{0}^{\pi} e^{i 2 \pi \alpha(\sin x \cos y)} \sin x d x d y=\operatorname{sinc}(2 \alpha),
$$

where $\operatorname{sinc}(x)=\frac{\sin (\pi x)}{\pi x}$.

Therefore, by using (43) and (44), Eqn. (40) can be expressed as

$$
\mathbb{E}\left\{\left|\boldsymbol{g}_{k}^{H} \boldsymbol{g}_{j}\right|^{2}\right\}=\beta_{k} \beta_{j} \chi_{k} \chi_{j}(M+\Omega),
$$

where $\Omega=\sum_{m=0}^{M-1} \sum_{n=0, n \neq m}^{M-1} \operatorname{sinc}^{2}\left(2(m-n) \frac{\delta}{\lambda}\right)$.

Finally, by substituting (45) and (39) into (36), we get the lower bound of ergodic capacity as given in (37).

\section{REFERENCES}

[1] M. Asadpour, B. Van den Bergh, D. Giustiniano, K. Hummel, S. Pollin, and B. Plattner, "Micro aerial vehicle networks: an experimental analysis of challenges and opportunities," IEEE Communications Magazine, vol. 52, no. 7, pp. 141-149, July 2014.

[2] T. Andre, K. Hummel, A. Schoellig, E. Yanmaz, M. Asadpour, C. Bettstetter, P. Grippa, H. Hellwagner, S. Sand, and S. Zhang, "Application-driven design of aerial communication networks," IEEE Communications Magazine, vol. 52, no. 5, pp. 129-137, May 2014.

[3] E. Yanmaz, R. Kuschnig, and C. Bettstetter, "Achieving air-ground communications in 802.11 networks with three-dimensional aerial mobility," in Proceedings of the IEEE International Conference on Computer Communications (INFOCOM), Apr. 2013, pp. 120-124.

[4] M. Asadpour, D. Giustiniano, K. A. Hummel, S. Heimlicher, and S. Egli, "Now or later?: Delaying data transfer in time-critical aerial communication," in Proceedings of the ACM Conference on Emerging Networking Experiments and Technologies (CoNEXT), Dec. 2013, pp. $127-132$.

[5] J. M. Abatti, Small Power: The Role of Micro and Small UAVs in the Future. Maxwell AFB, AL: Air Command and Staff College, 2005.

[6] P. Olsson, J. Kvarnstrom, P. Doherty, O. Burdakov, and K. Holmberg, "Generating UAV communication networks for monitoring and surveillance," in Proceedings of the International Conference on Control Automation Robotics Vision (ICARCV), Dec. 2010, pp. 1070-1077.

[7] F. Mohammed, A. Idries, N. Mohamed, J. Al-Jaroodi, and I. Jawhar, "UAVs for smart cities: Opportunities and challenges," in Proceedings of the International Conference on Unmanned Aircraft Systems (ICUAS), May 2014, pp. 267-273.

[8] T. L. Marzetta, "Noncooperative cellular wireless with unlimited numbers of base station antennas," IEEE Transactions on Wireless Communications, vol. 9, no. 11, pp. 3590-3600, Nov. 2010.

[9] E. G. Larsson, O. Edfors, F. Tufvesson, and T. L. Marzetta, "Massive MIMO for next generation wireless systems," IEEE Communications Magazine, vol. 52, no. 2, pp. 186-195, Feb. 2014.

[10] H. Q. Ngo, E. G. Larsson, and T. L. Marzetta, "Energy and spectral efficiency of very large multiuser MIMO systems," IEEE Transactions on Communications, vol. 61, no. 4, pp. 1436-1449, Apr. 2013.

[11] M. Asadpour, D. Giustiniano, and K. A. Hummel, "From ground to aerial communication: Dissecting WLAN 802.11N for the drones," in Proceedings of the ACM International Workshop on Wireless Network Testbeds, Experimental Evaluation \& Characterization (WiNTECH), Sept. 2013, pp. 25-32.

[12] M. A. Rahman, "Enabling drone communications with WiMAX technology," in Proceedings of the International Conference on Information, Intelligence, Systems and Applications (IISA), July 2014, pp. 323 328.

[13] J. Rasool, G. E. Øien, J. E. Håkegård, and T. A. Myrvoll, "On multiuser MIMO capacity benefits in air-to-ground communication for air traffic management," in Proceedings of the International Symposium on Wireless Communication Systems (ISWCS), Sept. 2009, pp. 458462.

[14] C. A. Balanis, Antenna Theory: Analysis and Design. WileyInterscience, 2005.

[15] M. Shafi, M. Zhang, A. Moustakas, P. Smith, A. Molisch, F. Tufvesson, and S. Simon, "Polarized MIMO channels in 3-D: models, measurements and mutual information," IEEE Journal on Selected Areas in Communications, vol. 24, no. 3, pp. 514-527, Mar. 2006.

[16] S. Jaeckel, K. Borner, L. Thiele, and V. Jungnickel, "A geometric polarization rotation model for the 3-D spatial channel model," IEEE Transactions on Antennas and Propagation, vol. 60, no. 12, pp. 59665977, Dec. 2012.

[17] M.-T. Dao, V.-A. Nguyen, Y.-T. Im, S.-O. Park, and G. Yoon, "3D polarized channel modeling and performance comparison of MIMO antenna configurations with different polarizations," IEEE Transactions on Antennas and Propagation, vol. 59, no. 7, pp. 2672-2682, July 2011. 